\title{
Emission of Type II Radio Bursts - Single-Beam versus Two-Beam Scenario
}

\author{
U. Ganse $^{1} \cdot$ P. Kilian ${ }^{1} \cdot$ R. Vainio ${ }^{2}$. \\ F. Spanier ${ }^{1}$
}

\begin{abstract}
The foreshock region of a CME shock front, where shock accelerated electrons form a beam population in the otherwise quiescent plasma is generally assumed to be the source region of type II radio bursts. Nonlinear wave interaction of electrostatic waves excited by the beamed electrons are the prime candidates for the radio waves' emission.

To address the question whether a single, or two counterpropagating beam populations are a requirement for this process, we have conducted $2.5 \mathrm{D}$ particle in cell simulations using the fully relativistic ACRONYM code.

Results show indications of three wave interaction leading to electromagnetic emission at the fundamental and harmonic frequency for the two-beam case. For the single-beam case, no such signatures were detectable.
\end{abstract}

Keywords: Radio Bursts, Type II; Radio Bursts, Theory; Plasma Physics

\section{Introduction}

Solar radio bursts have been observed since the earliest days of astronomical radio observations. Based on spectral morphology and development timescales, they are classified into 5 types (Wild and McCready, 1950). While suitable models for the emission of most types of radio bursts have since been found, an explanation of type II, and to some extent type III radio bursts' emission mechanisms remains elusive.

The morphology of type II bursts typically shows a two-band emission spectrum, consisting of the fundamental emission band and of the harmonic emission band at about twice the frequency of the fundamental (in a few, near-limb events signals of third harmonic emission are also discernable, see Zlotnik et al., 1998). The fundamental frequency is believed to correspond to the plasma frequency of the emission region, slowly decreasing over time as the coronal/interplanetary shock travels outwards into the heliosphere. (Cane. Sheeley, and Howard, 1987; Nelson and Melrose, 1985)

\footnotetext{
${ }^{1}$ Lehrstuhl für Astronomie, Universität Würzburg, Germany email: uganse@astro.uni-wuerzburg.de

2 Department of Physics, University of Helsinki
} 


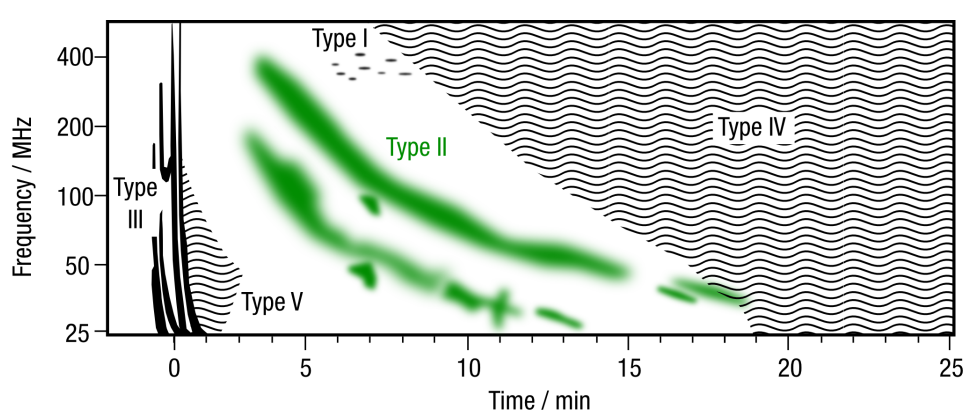

Figure 1. Exemplary classification of solar radio bursts by their morphology in a dynamic spectrum. Type II bursts are characterized by their two-banded emission of fundamental and harmonic frequency, which is slowly drifting towards lower frequencies.

Statistical studies have provided firm evidence of a correlation between type II bursts and the propagation of shock fronts outwards from the sun

(Nelson and Melrose, 1985; Forbes et al., 2006). These are in many cases driven by coronal mass ejections (CME), but have also been observed as blast waves from flare energy release (H. Aurass, B. Vrsnak, and G. Mann, 2002). It is therefore reasonable to assume that electron processes at the shock are responsible for the emission (Mann, 1995). At the time of radio emission, a CME shock front can span from the low solar chromosphere outwards to multiple solar radii, thus encompassing a large number of density scales and hence, potential plasma frequencies (Pomoell, Vainio, and Kissmann, 2008). The observed radio emissions, on the other hand, are quite narrowband. Consequently, the emission region can not be spread out along a large area of the shock front, but is rather required to have a small spatial scale, confined to a small subset of the shock (Schmidt and Gopalswamy, 2008). The emission region is thus expected to have special properties setting it apart from the rest of the shock.

\subsection{Type III Bursts}

Very similarly to type II bursts, the so-called type III bursts also feature multibanded emission, albeit with a much faster drift towards low frequencies and hence shorter total burst duration.

For these bursts, conclusive observational evidence exists for a connection to impulsive electron release in solar flares. As the accelerated electrons travel outwards on open field lines, they form an electron beam population within the solar wind plasma, which can excite Langmuir waves through Cherenkov-type instabilities (Karlickv́ and Vandas, 2007; Tsiklauri, 2010).

These Langmuir waves $(L)$ are then assumed to take part in three wave interaction processes (Melrose, 1986), leading to emission of transverse electromagnetic waves $(T)$ and sound waves $(S)$ :

$$
\begin{aligned}
L & \rightarrow L^{\prime}+S \\
L & \rightarrow S+T\left(\omega_{p e}\right) \\
L+L^{\prime} & \rightarrow T\left(2 \omega_{p e}\right)
\end{aligned}
$$



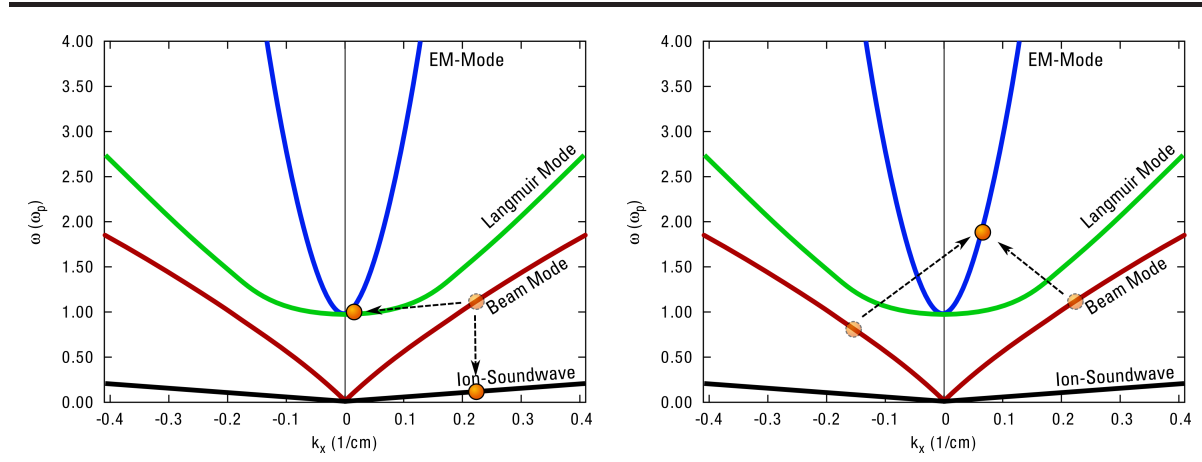

Figure 2. Sketch of three wave interaction processes believed to be the origin of fundamental (left) and harmonic (right) emission of type II radio bursts. Decay of beam-driven longitudinal waves into sound-waves and electromagnetic waves at the plasma frequency akin to the normal plasma emission process is responsible for the fundamental emission, whereas a coalesce process of two counter-directed modes leads to harmonic emission.

In a more detailed analysis, Willes and Cairns (2000) treated plasma wave behaviour in the presence of a beam population, and identified not the Langmuir wave, but the so-called beam driven mode as a possible source of three-wave interactions. As the beams' intensity decreases, energy in this mode would be transferred into the background plasma's eigenmodes, thus leading to similar processes as outlined in eq. 1,3, illustrated in figure 2,

Experimental proof of these processes has been challenging: satellite data (Pulupa and Bale, 2008), being intrinsically point-wise, can not yield information about the momenta and energies of participating waves in three-waves interactions.

While numerical investigation of three-wave interaction has been successfully performed using MHD codes (Spanier and Wisniewski, 2011), that technique is not applicable to the kinetic waves participating in the interactions outlined here. Particle-in-cell codes on the other hand, which are the tool of choice for kinetic simulations, have been too numerically expensive to be able to treat this problem self-consistently in the past. With the exponential growth of computing power, the length- and timescales of these emission models are coming into the range of top-end supercomputers now.

\section{Emission Model}

Due to the similarity to type III bursts in spectral shape, it seems reasonable to assume that electron beams are likewise responsible for type II radio emission. Assuming that the electron acceleration on the shock front is localized to a specific magnetic field configuration also elegantly solves the problem of the emissions' narrow-bandedness.

In the predominant model, Holman and Pesses (1983) proposed that electron shock drift acceleration on a curved shock front is the source of these electron beams. Following the interplanetary field from the point where the shock is exactly perpendicular into the foreshock plasma, these electron beams then excite 


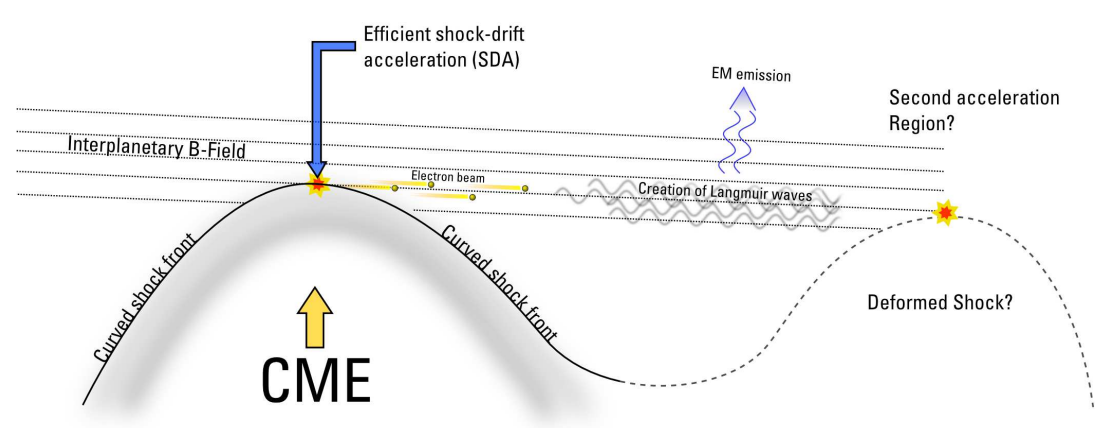

Figure 3. Sketch of the large scale model of the proposed emission process of type II radio bursts. Electrons accelerated through shock drift acceleration form a beam population in the foreshock plasma, exciting Langmuir waves which then undergo three-wave interactions. A variant of this model allows for multiple sites of electron acceleration on a deformed shock (such as a shock ripple) injecting counterstreaming electron beams into the same region.

Langmuir waves and undergo three-wave interactions much like eq. 1. 13 predicts for type III bursts. Since the efficiency of drift acceleration increases sharply around the sites where the shock angle is close to $90^{\circ}$, the source region of the electron beams is confined to a small area on the curved shock, thus explaining the narrowband nature of the emission.

An open question in these models remains whether the process of equation 1 itself - that is the decay of a forward-directed Langmuir wave into a forwarddirected sound wave and a backward-directed Langmuir wave - is sufficient to produce a large seed population of counterpropagating Langmuir waves for the process of equation 3 to produce significant intensities of radio emission at twice the plasma frequency. Alternatively, counterstreaming electron beams, directly exciting counterpropagating Langmuir waves may be required, as depicted in figure 3. These might be created in the case of shock ripples, or deformations of a shock due to shock-shock interaction, as reported multiple times in cases of very strong type II bursts.

\section{Numerical Simulation}

In order to decide whether a two beam scenario is required for harmonic emission, or whether a single-beam scenario is sufficient, we have conducted simulations using the ACRONYM particle in cell code.

\subsection{ACRONYM Code}

The ACRONYM code, developed and maintained at the department of Astronomy, University of Würzburg, is a fully relativistic, 2nd order particle-incell code for astronomical, heliospheric and laboratory plasmas. Using MPIparallelization, the code is running on all major supercomputer platforms (Kilian, Burkart, and Spanier, 2012).

For the simulations in this project, computing time on Jugene (BlueGene/P) at the Jülich Supercomputing Centre and Louhi (Cray XT-5) at the CSC Helsinki 


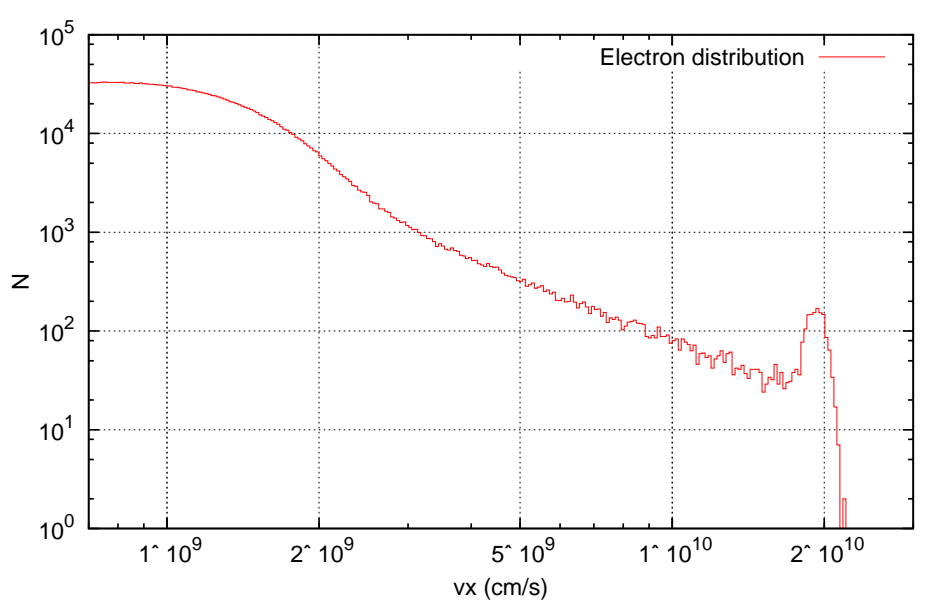

Figure 4. Electron velocity $\left(v_{x}\right)$ distribution at the start of a simulation in the single-beam case. A second, symmetric beam is included in the two-beam case

was employed. The runs took a total of about 1 Mio. CPU-hours on these machines.

\subsection{Setup}

Since a particle in cell code carries the intrinsic constraint of resolving the plasma debye length (in the case of solar wind, $\lambda_{D} \approx 1 \mathrm{~cm}$ ), the maximum size of a simulation domain is severely limited to the resolution of plasma-microphysical phenomena. A simulation of the complete shock environment depicted in figure 3 would be prohibitively expensive in terms of computing power. The simulations rather focus on the beam-plasma interaction and resulting three-wave processes in the foreshock plasma.

The simulations are set up as $2.5 \mathrm{D}$ (2D3V) rectangular grids with periodic boundaries, which are homogeneously filled with the background foreshock plasma under quiescent solar wind conditions $\left(T \approx 0.5 \mathrm{MK}, \rho=2.5 \cdot 10^{7} \mathrm{~cm}^{-3}\right.$, $B=1 \mathrm{G}$ ), with a thermal particle distribution. On top of that, either a single or two counterstreaming beamed electron populations are added at $v_{\text {Beam }} \approx 5 v_{\text {th }}$, whose density is about $10 \%$ of the total electron density (see fig. (4). The beam density and velocity is derived from typical Type II emission region parameters given in Knock et al. (2001), their pitch angle distribution is centered around $45^{\circ}$, following Karlický and Vandas (2007).

In the two-beam case, the simulation will be naturally charge-neutral and have a zero total current. In the single-beam case however, the background electron population is suitably boosted in the opposite direction to create an appropriate return current, and thus also zero out the total current within the simulation box. 
Table 1. Simlation parameters for the particle in cell simulation

\begin{tabular}{lcl}
\hline Physical parameters & & \\
\hline Background plasma density & $\rho$ & $2.5 \cdot 10^{7} \mathrm{~cm}^{-3}$ \\
Temperature & $T$ & $480280 \mathrm{~K}$ \\
Background magnetic field & $B_{x}$ & $1 \mathrm{G}$ \\
Plasma frequency & $\omega_{p}$ & $2 \cdot 10^{8} \mathrm{Rad} / \mathrm{s}$ \\
Beam density & $\rho_{B}$ & $0.1 * \rho$ \\
\hline Numerical parameters & & \\
\hline Box size in cells & $N_{x}$ & 8192 \\
& $N_{y}$ & 4096 \\
Cell size & $\Delta x$ & $0.95 \mathrm{~cm}$ \\
Timestep & $\Delta t$ & $1.2 \cdot 10^{-11} \mathrm{~s}$ \\
Particles per Cell & $n$ & 200 Background +20 Beam \\
\hline
\end{tabular}
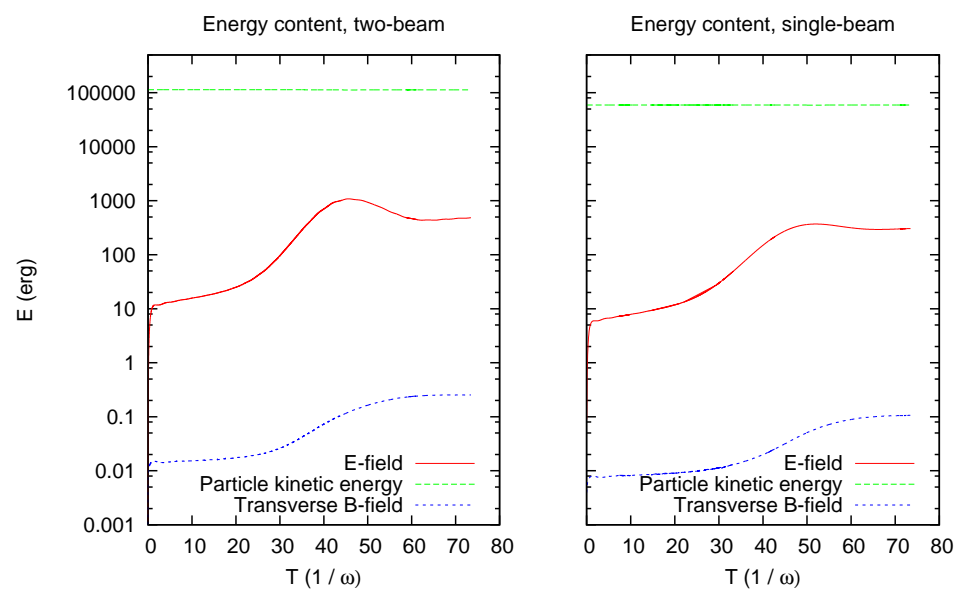

Figure 5. Energy distribution within two-beam (left) and single-beam (right) simulations, over time. After about 20 plasma timescales $\left(\omega_{p}^{-1}\right)$, the beam instability causes an increase in electric field energy. Subsequently, the transverse magnetic field energy also rises. (Background B-Field intensity is not shown, as it is constant throughout the simulation)

\section{Results}

Since the simulation is started with a quiescent thermal background plasma, into which the electron beam populations are injected, it takes a while (about $t=20 \omega_{p}^{-1}$ ) for the beam-driven instability to create a suitable amount of wave intensity. This can be easily observed by looking at the energy distribution graphs of single- and two-beam simulations, which are shown in figure 5.

In this graph, total particle kinetic energy, electric field energy and the energy content of the transverse magnetic field components is displayed over simulation 


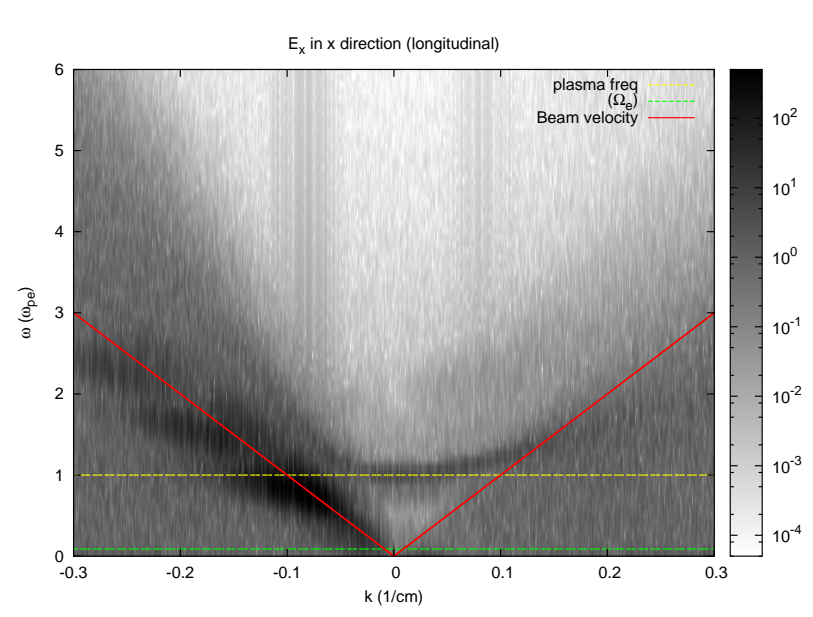

Figure 6. Dispersion plot of the longitudinal electric field component along the beam direction in a one-beam simulation. A strong excitation of the beam-driven mode, around resonance with the plasma frequency is visible in both forward direction (left).

time (the background magnetic field component is left out, since it is constant in good approximation).

The total particle kinetic energy contains a large contribution from the background plasma's thermal motion, making up about $61 \%$ of its value at the start of the simulation. The remaining $38 \%$, or $4.3 \cdot 10^{4} \mathrm{erg}$ (calculated from the particles' initial velocities) are contribution of the beam kinetic energy.

The electric field energy reaches a maximum at $t \approx 50 \omega_{p}^{-1}$, with a coinciding rise in energy in the transverse magnetic fields, implying an energy transfer from electrostatic to electromagnetic modes.

Confirming the results of Karlický and Vandas (2007), a peak electric field energy of about $2.8 \%$ of the beam kinetic energy is observed in both simulations. The energy content in the transverse magnetic fields at the end of the simulation runs lies about 3 orders of magnitude below that of the electric fields.

\subsection{Single-Beam Scenario}

In order to obtain quantitative information about the intensities of individual wave modes within the simulation, electric and magnetic field quantities were Fourier-transformed in spatial and temporal dimensions. The results are plotted as $k$ vs. $\omega$ intensity plots, in which each wave mode can be identified via its characteristic dispersion relation.

Figure 6 shows this dispersion plot for the longitudinal electric field component $\left(E_{x}\right)$ along the beam direction, with $k \| \vec{B}_{0}$. As expected, electrostatic longitudinal wave modes are discernible in this plot. In addition to the Langmuir wave, which is forming a parabolic shape around the plasma frequency at $k=0$, an additional, strong area of intensity is visible at negative $k$ values. The dispersion relation of this wave corresponds to a beam driven mode of the electron beam, which excites oscillations in the electron beam's density near resonance with the plasma frequency (Willes and Cairns, 2000). 


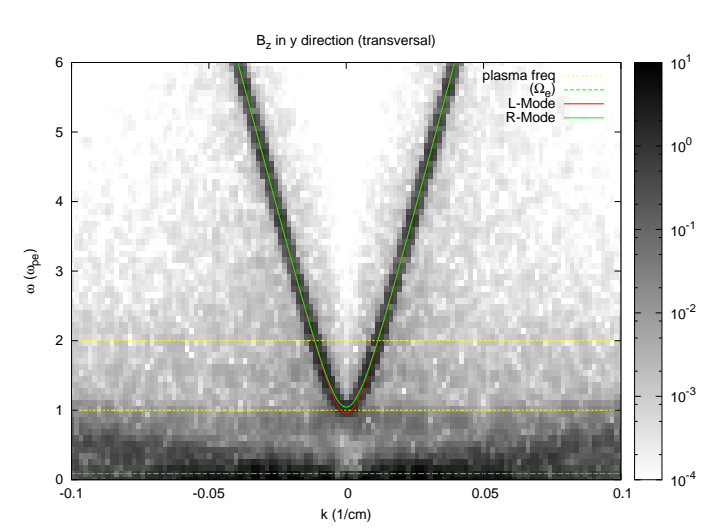

Figure 7. Dispersion plot of a transverse magnetic field component in the single-beam simulation, with $k$ perpendicular to the beam direction. The electromagnetic mode is clearly visible as a parabola, with a cutoff at the plasma frequency (splitting into L- and R-Mode is not sufficiently numerically resolved). No additional unexpected wave modes are observed.

By taking the sum of the energy content in the forward-propagating half space and comparing with the with the backward-propagating one, the beam driven modes' energy can be determined to $E_{f}=475 \mathrm{erg}$, whereas the backward direction only contains $E_{b}=0.3 \mathrm{erg}$ of energy. Note however that the energy content determined from this dispersion plot only contains modes which are exactly axisparallel (up to numerical precision) and is therefore grossly underestimating the beam-modes complete energy contribution (compare figure 5).

In figure 7 a similar dispersion plot for a transverse magnetic field component is shown. Apart from some thermal background excitation of the electromagnetic mode, no unexpected features are apparent in this plot. Especially, no indications of three-wave interaction are visible here.

\subsection{Two-Beam Scenario}

The longitudinal electric field dispersion plot for the two-beam case (figure 8) shows a symmetric structure of two counterpropagating beam-driven mode peaks, similar to figure 6. Additionally, a somewhat weaker feature is visible around $2 \omega_{p}$, which may be indicative of the three-wave coupling processes outlined in section 1.1 .

The dispersion plots of transverse magnetic field components (figure 9) show the expected dispersion relation of the electromagnetic mode, as predicted by linear theory. Additional bands of intensity at the fundamental and harmonic emission frequency are also visible. Consistent with the predicted processes outlined in section 1.1, these bands are likely created by nonlinear interaction of beam-driven waves with opposite direction of propagation.

For most $k$-values, the nonlinear product wave does not match an eigenmode of the background plasma and is hence unable to excite a propagating wave, explaining the low intensity of the feature in the dispersion plot. For $k$ values matching the electromagnetic mode however, electromagnetic waves can be resonantly excited, and travel through the plasma. 


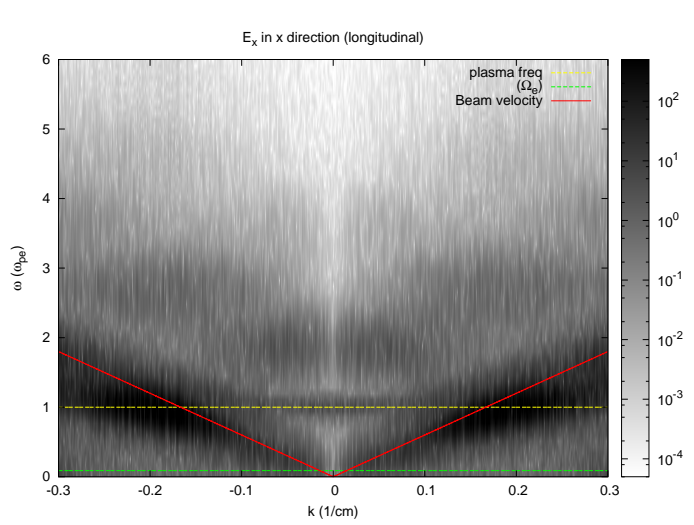

Figure 8. Dispersion plot of the longitudinal electric field component along the beam direction in a two-beam simulation. A strong excitation of the beam-driven mode, around resonance with the plasma frequency is visible in both forward and backward direction. Additionally, weaker but significant excitation appears to be present around $2 \omega_{p}$

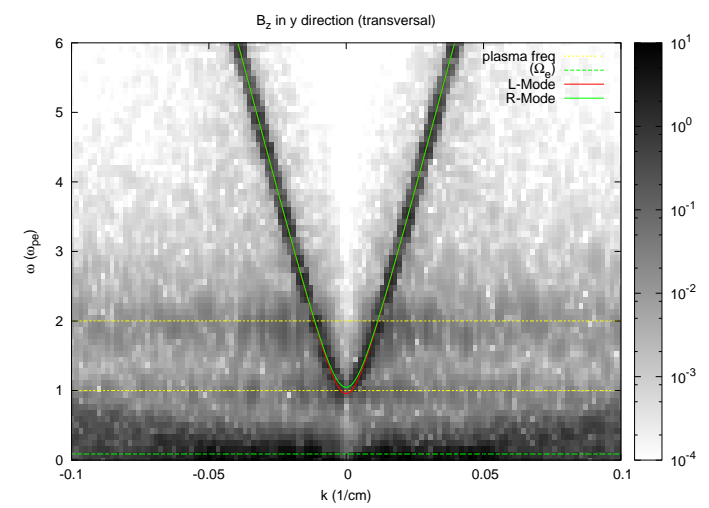

Figure 9. Dispersion plot of a transverse magnetic field component in the two-beam simulation, with $k$ perpendicular to the beam direction. The electromagnetic mode is clearly visible as a parabola, with a cutoff at the plasma frequency (splitting into L- and R-Mode is not sufficiently numerically resolved). In addition, two broad features at the fundamental and harmonic frequency are visible, which are in resonance with the em-Mode. (Intensities in arbitrary units)

\section{Conclusion}

We have compared particle in cell simulations of two different microphysical emission scenarios of type II radio bursts in a CME foreshock settings: one with a single electron beam accelerated by the shock, and another with two counterstreaming shock populations of equal strength.

While the single-beam scenario has shown production of electrostatic waves through beam-driven instabilities which are in line with theoretical predictions, emission of electromagnetic waves with fundamental and harmonic frequencies which would be the fingerprint of a type II burst, have not been observed.

In the two-beam simulation, electrostatic modes are likewise excited, leading to counterpropagating wave populations. Here, indications of nonlinear wave in- 
teraction are visible in the dispersion diagrams of transverse magnetic field component, with maxima at the plasma frequency and its first harmonic, conforming to the theoretically predicted emission process of type II bursts.

The results presented here should not be taken as a literary proof that a multi-beam environment is a necessity for type II emission: The canonical process to create counterpropagating wave populations for subsequent interaction is scattering of Langmuir waves on sound waves (eq. 11). By using a collisionless particle in cell approach for our simulations, we have completely neglected the effects of sound waves on the plasma, thus likely creating somewhat unrealistic wave dynamics.

Furthermore, due to the large numerical demands of the particle in cell simulation, the total physical simulation time was limited to a few microseconds. It is therefore possible that the electrostatic wave population has not relaxed into an equilibrium condition by the end of the run, such that scattering effects may have been underrepresented.

Still, the demonstrated strong dependence of harmonic radio emissions on the existence of counterpropagating electrostatic waves confirms long-standing theoretical predictions. In plasma environments where this mechanism is of importance, typical damping time of these waves has to be much larger than scattering times if a single beam mechanism is to be responsible for the radio emissions.

\subsection{Outlook and further work}

In this work, only the two extreme cases of a single beam and counterpropagating of equal strength beams have been investigated. In an actual type II emission region, beams will likely have different strengths and pitch angle distributions if they are originating from separate acceleration regions. The next step will therefore be simulations with varying beam intensities and angles.

The parameter space of these simulations further consists of the density / plasma frequency of the ambient plasma, magnetic field strength and temperature. Given sufficient computing time, parameters studies in this space may follow suit.

Acknowledgements The authors would like the Jülich Supercomputing Centre and the CSC Helsinki for their grants of computing time. UG and PK acknowledge financial support by the Elite Network of Bavaria. FS acknowledges support by the Deutsche Forschungsgemeinschaft, Grand SP1124-1. This work has been supported by the European Framework Programme 7 Grant Agreement SEPServer - 262773

\section{References}

Cane, H.V., Sheeley, J., Howard, R.A.: 1987, Energetic interplanetary shocks, radio emission, and coronal mass ejections. J. Geophys. Res. 92(A9), 9869-9874. doi 10.1029/JA092iA09p09869 http://dx.doi.org/10.1029/JA092iA09p09869. 
Forbes, T., Linker, J., Chen, J., Cid, C., Kta, J., Lee, M., Mann, G., Mikic, Z., Potgieter, M., Schmidt, J., Siscoe, G., Vainio, R., Antiochos, S., Riley, P.: 2006, Cme theory and models. Space Science Reviews 123(1), 251-302. http://dx.doi.org/10.1007/s11214-006-9019-8.

H. Aurass, B. Vrsnak, G. Mann: 2002, Shock-excited radio burst from reconnection outflow jet? A $\because A$ 384(1), 273-281. doi 10.1051/0004-6361:20011735 http://dx.doi.org/10.1051/00046361:20011735.

Holman, G.D., Pesses, M.E.: 1983, Solar type II radio emission and the shock drift acceleration of electrons. ApJ 267, 837 - 843. doi 10.1086/160918.

Karlický, M., Vandas, M.: 2007, Shock drift electron acceleration and generation of waves. Planet. Space Sci. 55, 2336-2339. doi 10.1016/j.pss.2007.05.015

Kilian, P., Burkart, T., Spanier, F.: 2012, The influence of the mass ratio on particle acceleration by the filamentation instability. In: Nagel, W.E., Krner, D.B., Resch, M.M. (eds.) High Performance Computing in Science and Engineering '11, Springer, Berlin Heidelberg, 513. ISBN 978-3-642-23869-7. doi 10.1007/978-3-642-23869-7 http://dx.doi.org/10.1007/9783-642-23869-7.

Knock, S.A., Cairns, I.H., Robinson, P.A., Kuncic, Z.: 2001, Theory of type II radio emission from the foreshock of an interplanetary shock. Journal of Geophysical Research 106, 25041 25052. doi $10.1029 / 2001 J A 000053$

Mann, G.: 1995, Theory and observations of coronal shock waves. In: Benz, A., Krger, A. (eds.) Coronal Magnetic Energy Releases Lecture Notes in Physics 444, Springer Berlin / Heidelberg, ???, 183-200. 10.1007/3-540-59109-5_50. ISBN 978-3-540-59109-2. http://dx.doi.org/10.1007/3-540-59109-5_50.

Melrose, D.B.: 1986, Instabilities in Space and Laboratory Plasmas, Cambridge University Press, Cambridge, UK..

Nelson, G.J., Melrose, D.B.: 1985, 7. Type II bursts, Cambridge University Press, ???, $333-$ 359.

Pomoell, J., Vainio, R., Kissmann, R.: 2008, Mhd modeling of coronal large-amplitude waves related to cme lift-off. Solar Physics 253(1), 249-261. http://dx.doi.org/10.1007/s11207008-9186-8.

Pulupa, M., Bale, S.D.: 2008, Structure on interplanetary shock fronts: Type ii radio burst source regions. Astrophysical Journal 676, 1330-1337.

Schmidt, J.M., Gopalswamy, N.: 2008, Synthetic radio maps of cme-driven shocks below 4 solar radii heliocentric distance. Journal of Geophysical Research 113, A08104.

Spanier, F., Wisniewski, M.: 2011, Simulation of Charged Particle Diffusion in MHD plasmas. Astrophysics and Space Sciences Transactions 7, 21-27. doi 10.5194/astra-7-21-2011

Tsiklauri, D.: 2010, Particle-in-cell, self-consistent electromagnetic wave emission simulations of type iii radio bursts. Solar Physics.

Wild, J., McCready, L.: 1950, Observations of the spectrum of high-intensity solar radiation at metre wavelengths. Australian Journal of Scientific Research 3(3), 387-398.

Willes, A.J., Cairns, I.H.: 2000, Generalized Langmuir waves in magnetized kinetic plasmas. Physics of Plasmas 7, 3167-3180. doi 10.1063/1.874180

Zlotnik, E.Y., Klassen, A., Klein, K.-L., Aurass, H., Mann, G.: 1998, Third harmonic plasma emission in solar type II radio bursts. A\&A 331, 1087-1098. 
SOLA: paper_arxiv.tex; 18 July 2018; 13:16; p. 12 